\title{
Die Anfänge der Wiener Handelsgerichtsbarkeit im 18. Jahrhundert
}

\author{
The Origins of Commercial Jurisdiction in Vienna in the $18^{\text {th }}$ Century
}

To promote international trade and in order to guarantee legal security for the local traders, the Austrian Emperor introduced a separate court for commerce-related cases in Vienna in 1717. This article explores the reasons for this act as well as the further developments up to the end of the $18 t^{\text {h }}$ century.

Keywords: Commercial jurisdiction - international trade - Vienna, $18^{\text {th }}$ Century

\section{Gerichtsorganisation Anfang des 18. Jahrhunderts und frühe Projekte zur Einführung einer Handelsgerichtsbarkeit}

Die Gerichtslandschaft in Wien im frühen 18. Jahrhundert war von einer großen Zersplitterung geprägt. An städtischen Gerichten bestanden das kaiserliche Stadt- und Landgericht und der Magistrat. Ersteres übte die Gerichtsbarkeit in gewissen Straf- und Zivilsachen aus, sofern die Angeklagten oder Streitparteien nicht besonderen Gerichten unterstanden. Es war etwa der ordentliche Gerichtsstand für bürgerliche Streitigkeiten der Wiener Bürger. Der Magistrat hingegen war das grundherrliche Gericht der Stadt, außerhalb des Burgfriedens der Stadt waren dies um Wien die anderen lokalen Grundherren. Daneben existierten Gerichte mit persönlicher Zuständigkeit. Zu nennen sind hier das akademische Gericht der Universität für ihre Angehörigen und das landmarschallische Gericht für den höheren Landadel. Gerichtsbarkeit hatten ebenfalls einige Stellen der herrschaftlichen Verwaltung, nämlich das Hansgrafenamt für Viehhändler, das Spielgrafenamt für Musikanten und das Wassergrafenamt für Fuhrleute,
Schiffer und Fischer. Zu erwähnen sind freilich auch die Militärgerichte sowie die geistlichen Gerichte Wiens. In erster und zweiter Instanz war zudem die niederösterreichische Regierung tätig, etwa in landgerichtlichen Sachen. ${ }^{1}$

Kaufleute waren in Wien je nach Stand und Herkunft vor Einführung des Wechselgerichts drei verschiedenen Gerichten unterworfen. Für die sogenannten Niederleger war die niederösterreichische Regierung zuständig, für die Hofbefreiten das Hofmarschallamt und für die (handelnden) Bürger Wiens das Stadtgericht. ${ }^{2}$ Worum es sich bei den Bürgern Wiens handelt, ist klar; Hofbefreite waren Händler oder Handwerker, welche direkt für den Hofstaat des Kaisers agierten. ${ }^{3}$ Die Niederleger waren ausländische Kaufleute, welche in Wien ansässig waren und in großem Stil Fernhandel betrieben. Ihr Stand ging auf die Stapelrechte von 1221 und

\footnotetext{
${ }^{1}$ BARTSCH, Wiener Gerichte 117-123.

2 ÖStA, HKA, Alte Hofkammer, Pat. 16/57a, Kart. 48.13, Wechsel-Patent Begreiffend in sich Die Wechsel-Ordnung Dann auch Das Wechsel-Gericht Erst/anderter und letzter Instanz Wie es in ein und andern im Land Oesterreich unter der Ennß solle gehalten werden (1717), Abschnitt Wechselgericht $\S 1$. ${ }^{3}$ Perger, Haupt, Hofbefreite Handwerker 220.
} 
1312 zurück, denen zufolge fremde Kaufleute verpflichtet waren, ihre Waren in Wien „niederzulegen" und einheimischen Händlern anzubieten, wodurch der ausländische Direkt- und Weiterhandel bis auf wenige Ausnahmen ausgeschlossen war. In der frühen Neuzeit gewährte man den Niederlegern Freihandel mit bestimmten Mindestwarenmengen, wodurch sie eine starke wirtschaftliche Stellung erlangten, große Warenhäuser errichten konnten und aufgrund ihrer Kapitalkraft wesentliche Kreditgeber in der Stadt waren. Sie genossen religiöse Toleranz und mussten als Fremde außer Zöllen und Mauten keine Steuern zahlen. ${ }^{4}$ Die mit Handelssachen befassten Gerichte entschieden die Streitigkeiten nach dem Gewohnheitsrecht des Handelsortes oder aber nach dem anderer Handelsplätze. ${ }^{5}$

Die wirtschaftliche und rechtliche Situation der Händler in Niederösterreich dürfte Anfang des 18. Jahrhunderts eine sehr schwierige gewesen sein. Dies geht aus einem Gutachten des Wiener Handelsstandes an die kaiserliche BancoDeputation vom 5. April 1707 hervor. In diesem Gutachten legten die Vertreter des Wiener Handelsstandes die Gründe dar, warum ihrer Ansicht nach „der allgemeine Handl verfallen seye, [...] treu und glauben gleichsamb verschwunden und das Commercium gäntzlich darnieder lige". ${ }^{\prime}$ Im Zuge der weitschweifenden Ausführungen werden viele Probleme genannt. Erstens herrsche im Heiligen Römischen Reich seit etwa einem Jahrhundert fast unentwegt ein kriegerischer Zustand. Dieser beeinträchtige die allgemeine Sicherheit, welche der Handel benötige, und auch das Vertrauen unter den Händlern. ${ }^{7}$

\footnotetext{
${ }^{4}$ Perger, Niederleger 398f.

${ }^{5}$ WEIGAND, Handelsrechtsgesetzgebung 133.

${ }^{6}$ ÖStA, HKA, Alte Hofkammer, Vorschläge rote Nummer 8, Fasz. 8/2, Kart. 16, Gutachten der Deputierten der drei Handelsstände an die kaiserliche Banco-Deputation vom 5. 4. 1707 fol. 687 ${ }^{\mathrm{r}}$.

${ }^{7}$ Ebd. fol. 689v.
}

Schwerwiegender seien jedoch zweitens die Zersplitterung der Gerichtsbarkeit und die lange Dauer und die hohen Kosten der Prozesse zu werten. Die Kaufleute hätten unterschiedliche Gerichtsstände, wenn sie einander klagten und selbiges galt auch für etwaige nicht dem Handelsstand angehörende Schuldner. Die verschiedenen Gerichte wiederum gewährten verschiedene Einreden und ließen Berufungen zu, welche wiederum $\mathrm{zu}$ jeweils anderen Instanzen führten. So konnte es kommen, dass der Gläubiger mehrere Jahre hindurch bei verschiedenen Gerichten prozessieren musste und stets dem Prozessrisiko ausgesetzt war. Wegen der hohen Kosten für Anwälte und Gerichte hätten daher im Tagesgeschäft stehende Kaufleute oft davon abgesehen, kleine Beträge wie 30, 40 oder 50 Gulden einzuklagen, denn die Kosten überstiegen die eingeklagten Schulden bei weitem. Es war auch der Fall, dass durch Kostentragungsentscheidungen und Aufschiebung der Exekution der Kläger oftmals nicht nur diese nicht ganz ersetzt bekam, sondern auch länger auf die Bezahlung warten musste. ${ }^{8}$ Andere Ursachen waren die Schwankungen des Geldwertes, die mangelhafte Überwachung des Stapelrechts und der damit einhergehenden Strafbestimmungen, der Schmuggel und Schwarzhandel, welcher auch von Adeligen betrieben wurde, der Mißbrauch von Monopolprivilegien und schließlich das große „wachsthumb der allhiesigen Judenschafft", welches daher rühre, dass sich mit den unter kaiserlichem Schutz stehenden Faktoren, Händlern und Lieferanten auch viele andere Juden nach Wien begeben würden. ${ }^{9}$ Welchen Abbruch die Präsenz einer jüdischen Gemeinde dem allgemeinen Handel denn genau täte, führten die Gutachter freilich in ihren antisemitischen Anmerkungen nicht aus.

\footnotetext{
${ }^{8}$ Ebd. fol. 689v-691v.

${ }^{9}$ Ebd. fol. 691ㄴ-692v.
} 
Die Handelsleute boten einige Lösungsvorschläge an, von denen uns hier nur derjenige interessiert, welcher mit dem Handelsgericht und dem Wechselrecht zu tun hat. Es sei, so das Gutachten, zur Abhilfe gegen die Verfahrensprobleme in Handelssachen „kein ande[re]s Mittel als ein wohl eingericht [...] Judicium Mercantile", welches eine eigenständige Einrichtung sein sollte und eine zu erlassende „Kaufmanns- und Wechsel Ordnung" anzuwenden hätte. Es folgen Beispiele aus anderen Städten wie etwa Konstantinopel, Antwerpen, Hamburg, Nürnberg, Frankfurt, Leipzig, Breslau und Lübeck, wo die Handelsgerichtsbarkeit unterschiedlich ausgestaltet war, zum Teil jedoch schon autonome Tribunale und Standesgerichte für Handelsstreitigkeiten zuständig waren. Man verwies auf ein einige Jahre zurückliegendes Projekt des Handelsstandes, ein solches Gericht mit Exekutionsbefugnis einzuführen, das offenbar unter Zuziehung von mehreren Rechtsgelehrten erarbeitet worden war. ${ }^{10}$

Dieses Projekt dürfte mit dem im Österreichischen Staatsarchiv im selben Akt enthaltenen Entwurf für eine Wechselordnung aus der Regierungszeit Josephs I. identisch sein. Er stammt von Repräsentanten der drei Klassen des Wiener Handelsstandes und von zumindest einem Juristen namens Johann Georg Managetta. ${ }^{11}$ Die diesem leider nicht näher datierten Schriftstück vorgestellte Präambel gibt Aufschluss über die Gründe, welche für diesen angedachten Gesetzgebungsakt maßgeblich waren. Zunächst wird auf die Politik des Kaisers verwiesen, die auf eine generelle Förderung des Handels abzielte, und eine entsprechende Justiz sei „anima comercii et societatis civilis". ${ }^{12}$ Danach beschreibt man,

\footnotetext{
${ }^{10}$ Ebd. fol. 693 ${ }^{\mathrm{v}}-700^{\mathrm{r}}$.

${ }^{11}$ Ebd. fol. 685 ${ }^{\mathrm{r}}$. Siehe dazu schon, freilich nur im Hinblick auf die Protokollierungspflicht des zukünftigen Gerichts RINTELEN, Merkantilprotokoll 259-261.

12 ÖStA, HKA, Alte Hofkammer, Vorschläge rote Nummer 8, Faszikel 8/2, Kart. 16, Ohnmaßgebiger
}

welche Probleme konkret im Zusammenhang mit Wechseln auftraten: Zunächst waren es die Streitigkeiten, in denen Laien involviert waren, welche einen Schuldschein ausstellten, und nicht wussten, dass es ein Wechsel war und die damit verbundenen rechtlichen Wirkungen nicht kannten. Darüber hinaus gab es aber auch bei Streitigkeiten mit wechselkundigen Kaufleuten Schwierigkeiten, da die zuständigen Gerichte nicht wussten, ob sie einheimisches Gewohnheitsrecht oder das Recht eines fremden Wechselplatzes anzuwenden hatten. ${ }^{13}$ Im Entwurf gibt es einundfünfzig Artikel, welche Wechselgeschäfte und auch Konkurse regeln. Die Art. 4 und 51 beschäftigen sich mit einem Wechselgericht: Es sollen laut Art. 4 nämlich alle, die einen Wechselbrief ausstellen, seien sie männlich oder weiblich, geistliche oder weltliche Fürsten, Adelige, deren Bedienstete, Universitätsangehörige oder Militärpersonen, an die Wechselordnung gebunden und einem zu errichtenden „Mercantilgericht" unterworfen sein. Dies ist freilich eine totale Abkehr von der damals bestehenden fragmentierten Standesgerichtsbarkeit. Interessant ist die Marginalie im Entwurf, welche ergänzt, dies stimme mit dem allgemeinen Gebrauch („,communi usu cambiali“) und insbesondere mit der Leipziger und Berliner Ordnung überein. Es wird also auf deutsche Vorbilder zurückgegriffen. Art. 51 enthält weitere Ausführungen: Das Wechselgericht solle unabhängig sein, es solle nur eingeschränkte Berufungsmöglichkeiten geben, und im Falle eines Rechtsmittels sollte die Wechselsumme vom Rechtsmittelwerber bei Gericht $\mathrm{zu}$ hinterlegen sein. ${ }^{14}$ Interessanterweise ist im selben Bestand auch ein aus seinem Umfeld nicht ohne Probleme

Entwurf einer alhier in Wienn aufrichtender Wechselordnung fol. $665^{\mathrm{r}}$.

${ }^{13}$ Ebd. fol. 665v.

${ }^{14}$ Ebd. fol. 666 $-666^{\mathrm{v}}, 677^{\mathrm{v}}-678^{\mathrm{r}}$. 
kontextualisierbarer ${ }^{15}$ Entwurf einer Handelsgerichtsordnung enthalten, welcher in die Zeit Leopolds I. zurückreichen dürfte. Darin wird die Errichtung eines einheitlichen Gerichtes für alle Handelsstreitigkeiten angeordnet, welches aus sieben Handelsleuten - einem Richter und sechs Beisitzern - bestehen soll. Je zwei Beisitzer sollen von den Hofbefreiten, zwei von den Niederlegern und zwei von den bürgerlichen Händlern bestimmt werden, ohne die Religion oder Herkunft zu beachten. Die Rangordnung der Beisitzer war festgelegt, nämlich zuerst einer aus den Hofbefreiten, der zweite von den Niederlegern und der dritte von den Bürgern. Der Richter sollte aus einer der drei Klassen für ein Jahr gewählt werden, wobei danach die jeweils nächste Gruppe zum Zug kommen sollte. Zuständig sollte es ausschließlich sein für alle Streitigkeiten der Handelsleute untereinander, nicht aber für alle Streitigkeiten, an denen nur ein Kaufmann beteiligt war. In diesem Fall sollte man den Beklagten an seinem Gerichtsstand klagen. ${ }^{16}$ Um ein Urteil zu fällen sollten alle, zumindest aber vier Beisitzer zusammen mit dem Richter anwesend sein. Es wurde reihum abgestimmt, wobei die Reihenfolge Hofbefreite, Niederleger und bürgerliche Händler unter den Abstimmenden einzuhalten war, und zwar so, dass je die auf die Gruppe des Richters folgende Gruppe zuerst an die Reihe kam. Bei Stimmengleichheit gab die Stimme des Richters den Ausschlag. ${ }^{17}$ Weitere Bestimmungen regelten die Nachbesetzung der Stellen, die Tätigkeit der Gerichtsnotare, der Boten, die Zuziehung von Rechtsgelehrten, Konkurse („Fallimente“), ein-

\footnotetext{
${ }^{15}$ Laut einem eingelegten Stück Papier soll er einer handschriftlichen Notiz zufolge 1720 zur Kenntnis der aktenerzeugenden Stelle gelangt sein.

${ }^{16}$ ÖStA, HKA, Alte Hofkammer, Vorschläge rote Nummer 8, Fasz. 8/2, Kart. 16, undatierter und ungezeichneter Entwurf einer Handelsgerichtsordnung aus der Regierungszeit Leopolds I. (rote Notiz „Fasz. 1 Nr. 10“) fol. 703v-705v.

${ }^{17}$ Ebd. fol. $706^{\mathrm{r}}-706^{\mathrm{v}}$.
}

zelne Ausnahmen der Gerichtsbarkeit, die Arretierung von Verdächtigen, Zinsen und die Eidesablegung. ${ }^{18}$

Von Interesse ist an diesem Entwurf, dass es sich bei dem ins Auge gefassten Gericht schon um ein Handelsgericht handelt, welches nicht auf Wechselstreitigkeiten eingeschränkt ist. Gleichzeitig steht es jedoch auf dem Boden der althergebrachten Standesgerichtsbarkeit, weil es einen Gerichtsstand für Kaufleute schafft und die Streitigkeiten zwischen Kaufleuten und Nichtkaufleuten auf die bestehenden jeweiligen Gerichte für den Beklagten verweist. Damit unterscheidet es sich von den oben erwähnten, zeitlich danach anzusiedelnden Entwürfen, geht aber in Fragen der Gerichtsorganisation (Richter und Beisitzer aus den drei Klassen, alternierendes System) bereits den Weg, den die Wechselordnung von 1717 beschreiten sollte.

\section{Die Wechselordnung von 1717 und die Errichtung des Wechselgerichts in Wien}

Die eben besprochenen Pläne und Entwürfe kulminierten am 10. September 1717 in der Erlassung einer Wechselordnung für Österreich unter der Enns. Diese Wiener Wechselordnung sollte vornehmlich mit den Missständen aufräumen, die schon in der Präambel zum oben erwähnten Entwurf des Kaufmannsstandes aus der Zeit Josephs I. erwähnt worden waren, wie etwa die Rechtsunsicherheiten und die Streitigkeiten zwischen Kaufleuten und Nichtkaufleuten über das Vorliegen eines Wechsels. ${ }^{19}$ Sie sei, wie es in einem zeitgenössischen juristischen Kommentar heißt, „,nach dem Exempel anderer vornehmen Handels-Städte mit Zuziehung verständiger der Handels- und Wechsels-Sachen

\footnotetext{
${ }^{18}$ Ebd. fol. 705v $-706^{\mathrm{r}}, 706^{\mathrm{v}}-713^{\mathrm{v}}$.

${ }^{19}$ WeIGAND, Handelsrechtsgesetzgebung 134.
} 
wolerfahrner Personen“20 entstanden, was darauf hindeutet, dass die oben schon erwähnten „Lobbyisten" der drei Wiener Handelsklassen wohl einigen Einfluss auf das Projekt gehabt haben dürften. Neben einer detaillierten Regelung des Wechselrechts enthielt die Wechselordnung von 1717 zahlreiche Bestimmungen über ein $\mathrm{zu}$ errichtendes Wechselgericht. Im Folgenden soll das materielle Recht nur insofern behandelt werden, als es zum Verständnis der Gerichtsorganisation notwendig ist.

Zentraler Anknüpfungspunkt für die Zuständigkeit des Gerichtes war der Wechselbrief. Er war definiert als „eine Schriftliche Verbindnus vermög welcher der Ausgeber des WechselBriefs das auf einem Platz empfangene Geld dem Inhaber des Wechsel-Briefs auf einem anderen Platz in dem Wehrt nach bedungenen Wechsel-Lauf wiederum zuverschaffen und $\mathrm{zu}$ bezahlen zu lassen schuldig ist" Dieses Papier ließ sich in zwei Untergruppen unterteilen: Es gab Wechsel, welche gewisse formale Kriterien erfüllten und damit als „formige Wechsel“ galten. Sie mussten etwa den Ort, das Datum, den Zahlungstermin, die Art und Höhe der Rückzahlung und die Unterschriften der Parteien enthalten. ${ }^{21}$ Jede Person ohne Ansehung von Geschlecht, Stand oder Herkunft, welche sich solch eines „formigen Wechsels" bediente, war der Wechselordnung unterworfen, und Streitigkeiten aus den Wechseln mussten vor das Wechselgericht gebracht werden. Dabei gab es keine Ausnahmen. Die Kaufmannseigenschaft im modernen Sinne begründete eine Ausstellung eines solchen Wechsels jedoch nicht. ${ }^{22}$ Auch Juden waren nicht ausgenommen, wie aus dem oben zitierten zeitgenössischen Kommentar hervorgeht: „Hierbey könnte ein Zweifel entste-

\footnotetext{
${ }^{20}$ WEGELIN, Wechsel-Recht fol 2v.

${ }^{21}$ ÖStA, HKA, Alte Hofkammer, Pat. 16/57a, Kart. 48.13, Wechsel-Patent, Abschnitt Wechselordnung Art. 2.

22 Ebd. Art. 6.
}

hen, ob auch die Juden mit Recht den WechselHandel treiben, und auf Christen, \& vice versa diese auf jene, einen Wechsel-Brieff ziehen, oder indossiren können? Alldieweilen in denen gemeinen Kayserl. Rechten so wol als denen Reichs-Fundamental-Gesetzten [...] ausdrücklich verordnet ist, daß kein Jude von einem Christen eine Schuld kauffen, oder seine Schuld einem Christen bei Verlust der SchuldForderung cediren solle. Allein es bezeuget die tägliche Erfahrung, daß denen Juden mit denen Christen keines weges mehr verbotten sondern zu mehrerem Flor, Aufnahm und Beförderung der Commercien (da ihnen [...] ohne dem desselben Ursprung $u$. Erfindung einiger massen zugeschrieben wird) allerdings zugelassen sey den Wechsel-Handel zu treiben, zumalen ohnehin Handel und Wandel nicht fragt, noch fragen darff, ob der Negotiant, wenn er Treu und Glauben hält, ein Christ/Jud/Türck oder Heyde seye, die Juden auch sonsten heut zu Tag fast an den meisten Orten in Europa vollkommene Schutz-Gerechtigkeit geniessen. “23

Sogenannte „ohnformige Wechsel", welche die formalen Kriterien nicht erfüllten, brachten jedoch nicht ohne weiteres die Anwendbarkeit der Wechselordnung mit sich. Da solche Wertpapiere eher als Schuldscheine anzusehen waren, sollten Streitigkeiten, welche sich aus ihnen ergaben, nur zwischen Kaufleuten die Anwendbarkeit des Gesetzes und damit die Zuständigkeit des Wechselgerichts begründen. War die Streitigkeit jedoch zwischen einem Kaufmann und einem Nichtkaufmann, waren die herkömmlichen Gerichte zuständig und die Wechselordnung nicht anwendbar. ${ }^{24}$

In einem gesonderten Abschnitt regelte die Wechselordnung von 1717 die Organisation des Gerichts. Seine prinzipielle Zuständigkeit waren

\footnotetext{
${ }^{23}$ WeGELIN, Wechsel-Recht 25.

24 ÖStA, HKA, Alte Hofkammer, Pat. 16/57a, Kart. 48.13, Wechsel-Patent, Abschnitt Wechselordnung Art. 54.
} 
die Wechselstreitigkeiten nach den eben genannten Kriterien. Es bestand aus sieben „tauglichen, ehrbaren und wol-verständigen HandelsLeuten, als einem Richter und sechs Beysitzern“. ${ }^{25}$ Damit lag die Gerichtsbarkeit voll in der Hand der Kaufleute, womit einer der traditionellen Grundgedanken der Handelsgerichtsbarkeit verwirklicht war, nämlich, dass zur Beschleunigung und Sicherung der Qualität der Rechtsprechung eine Beteiligung von Fachleuten wünschenswert sei. ${ }^{26}$ Anfänge dafür lassen sich neben einigen Spuren im Mittelalter vor allem in einem Privileg von Kaiser Maximilian aus dem Jahr 1508 feststellen. Es sah ein Laienrichtertum und Verfahrensvereinfachungen für Handelsstreitigkeiten im Gegensatz $\mathrm{zu}$ den anderen Gerichten vor und lässt sich in Nürnberg und Leipzig zuerst nachweisen. ${ }^{27}$

Der Richter wurde aus einer der Klassen für zwei Jahre gewählt, und zwar zunächst aus der Klasse der Niederleger, dann für die nächsten zwei Jahre aus jener der Hofbefreiten und schließlich aus der Mitte der bürgerlichen Handelsleute, wonach sich diese Reihenfolge wiederholte. Jede Klasse wählte auch zwei Beisitzer, welche bis zur Resignation, Tod oder Abberufung ihren Dienst versahen. Alle diese mussten vom Kaiser bestätigt werden. Gewählt werden konnten erfahrene Kaufleute aus den diversen Sparten des Wiener Handels. Der Richter wurde nach Ablauf seiner Amtszeit Beisitzer. Das Gericht sollte wöchentlich zwei Mal am Montag und Donnerstag tagen, aber bei Gefahr im Verzug jederzeit zusammen gerufen werden können. Zur Fällung eines Urteils sollten der Richter und alle Beisitzer, zumindest jedoch der Richter und vier Beisitzer anwesend sein. Abgestimmt wurde einzeln in der Reihenfolge Niederleger-

\footnotetext{
${ }^{25}$ ÖStA, HKA, Alte Hofkammer, Pat. 16/57a, Kart. 48.13, Wechsel-Patent, Abschnitt Wechselgericht Titel $1 \S 1$.

${ }^{26}$ DusChKOW-KeSSIAKOFF, Handelsgericht 15.

${ }^{27}$ Ebd. 32f.
}

Hofbefreite-bürgerliche Händler, wobei die Gruppe als erste ihre Stimme abgab, welche nach jener des Richters kam. Bei einem Richter aus den Niederlegern stimmte etwa ein Beisitzer der Hofbefreiten, dann einer der bürgerlichen Händler und dann einer der Niederleger und so weiter ab. Der Richter stellte eine Stimmenmehrheit fest oder entschied bei Stimmengleichheit. ${ }^{28}$ Weitere Regelungen betrafen die Zuziehung von Rechtsgelehrten, die Tätigkeit der Gerichtsnotare, Ansager und Boten, die Urteilsfällung, Berufungsmöglichkeit und Exekutionsdurchführung, die Eide der Gerichtspersonen sowie noch einmal explizit die bereits in der Wechselordnung festgelegte Zuständigkeit. ${ }^{29}$

Als zweite Instanz in Wechselsachen sah man ein "Appellations-Wechsel-Gericht" vor, bestehend aus einem Richter aus dem Herrenstand und sechs „Mercantil-Räthen“, nämlich einem Rat der niederösterreichischen Regierung, einem obristhofmarschallischen Assessor, einem Stadtgerichtsassessor und je einem aus den drei Klassen des Handelsstandes. Dieses Gericht hatte nach Stimmenmehrheit umgehend $\mathrm{zu}$ entscheiden. ${ }^{30}$ Als dritte Instanz für Revisionen „zum Schutz und Schirm der Gerechtigkeit" war die Hofkanzlei zuständig. Die Berufungsmöglichkeit war allerdings eingeschränkt: Bei zwei gleichlautenden Urteilen der unteren Instanzen war sie nicht möglich, es sei denn der Revisionswerber konnte „erhebliche Ursachen“ vorbringen. Die Revision hatte schriftlich zu erfolgen, hinderte nicht die Exekution des Urteils zweiter Instanz und der Werber musste gegebenenfalls eine Sicherstellung des zuerkannten

${ }^{28}$ ÖStA, HKA, Alte Hofkammer, Pat. 16/57a, Kart. 48.13, Wechsel-Patent, Abschnitt Wechselgericht Titel $1 \S \S 2-4$.

${ }^{29}$ Ebd. Titel 1 §§ 5-9. In der Literatur wird gelegentlich erwähnt, dass „Geistliche und Militärpersonen“ ausgenommen gewesen seien, wobei das Gegenteil aus der Wechselordnung Art. 6 hervorgeht; vgl. BARTSCH, Wiener Gerichte 125; KAINRAD, Beiträge 6. ${ }^{30}$ Ebd. Titel 2 §§ 1-3. 
Betrages leisten. Den Parteien wird im Gesetz im Gegenzug eine Behandlung der Revision innerhalb eines Monats in Aussicht gestellt. ${ }^{31}$

\section{Die weitere Entwicklung des Wechselgerichts im 18. Jahr- hundert hin zu einer umfassen- den Handelsgerichtsbarkeit}

In den folgenden Jahren entfaltete das Wechselgericht in Wien seine Tätigkeit. Zu erwähnen ist in diesem Zusammenhang, dass es auch für die Entstehung des Handelsregisters eine zentrale Rolle spielte. Ihm war nämlich die Führung des sogenannten Merkantilprotokolles übertragen: Darin sollten gemäß Art. 8 der Wechselordnung von 1717 die Namen der Gesellschafter eines Unternehmens genannt werden, um bei Streitigkeiten oder Konkursen einen Anhaltspunkt zu haben. Dies scheint aber bis 1725 kaum beachtet worden zu sein, weswegen eine kaiserliche Deklaration aus diesem Jahr dies nochmals anordnete. Aus der Folgezeit sind dann auch solche Protokolle erhalten. ${ }^{32}$ Eine weitere Ausdehnung der Aufgaben erfolgte durch die „Fallitenordnung“ von 1734. Sie wurde aus Anlass zahlreicher Bankrotte erlassen und sah unter anderem vor, dass neue Unternehmen ihre Gesellschafter und die Höhe des Kapitals beim Gericht anzugeben hatten, um bei eventuellen Haftungen klare Informationen $\mathrm{zu}$ haben. Von großer Bedeutung war auch die Bestimmung der Fallitenordnung, dass bei Konkurs einer Unternehmung die Ehefrau nicht ohne weiteres die vorzügliche Befriedigung etwaiger Ansprüche aus einem Ehepakt fordern konnte, sofern die Konkursmasse nicht ausreichte, um alle Gläubiger zu befriedigen. Auch mussten neue Eheverträge dem Wechselgericht zur Kenntnis-

\footnotetext{
${ }^{31}$ Ebd. Titel 3 §§ 1-4.

32 RINTELEN, Merkantilprotokoll 265f, 275-281.
}

nahme und gegebenenfalls Approbation vorgelegt werden. Diese beiden Vorschriften dürften nicht volle Beachtung durch die Kaufmannschaft gefunden haben und so musste sie 1755 durch eine Hofresolution wieder eingeschärft werden. ${ }^{33}$

Im Zuge der großen Reformen Maria Theresias blieb das Wechselgericht nicht verschont. Um die „vielen Gerichtsstellen und weitschichtige Gerichtsordnung" einzuschränken, hob man neben einigen anderen Sondergerichten auch das Wechselgericht in Wien 1749 auf.34 Die Agenden wurden bei der niederösterreichischen Regierung konzentriert. Im Laufe der folgenden Jahre zeigte sich aber, dass sich dies nicht bewährte. Als im Jahre 1763 eine neue Wechselordnung erlassen wurde, stellte man gleichzeitig mit Resolution die Wechselgerichte erster und zweiter Instanz per 1 . November wieder her. ${ }^{35}$

Die Wechselordnung vom Oktober 1763 war für das Wiener Wechselgericht besonders bedeutsam. Sie sah die oben erwähnten Gerichte vor und enthielt Bestimmungen zu deren Organisation. ${ }^{36}$ Sie war inhaltlich an die Wechselordnung von 1717 angelehnt, ging aber im Regelungsgehalt und im Umfang weit über diese hinaus. Zentraler Anknüpfungspunkt blieb der Wechsel, der Wirkungskreis der „Wechsel- und Merkantilgerichte" wurde jedoch bedeutend ausgeweitet: "Zu dem Wechselgerichte gehören alle Streitsachen, so theils von förmlichen, trockenen und unterworfenen Wechselbriefen entspringen, theils aber zwischen beiderseitigen Negozianten, wohin auch die Fabriksgesellschaften, in Handlungssachen gehörig, über allerhand Merkantil- und Handlungsgeschäfte erwachsen, und

\footnotetext{
${ }^{33}$ KAINRAD, Beiträge 9f; RINTELEN, Merkantilprotokoll 269-275.

${ }^{34}$ BANNIZA, Vollständige Abhandlung 43; BARTSCH, Wiener Gerichte 125; DOMIN-PETRUSHEVECZ, Rechtsgeschichte 35 .

35 WALDSTÄTTEN, Gerichte 35.

${ }^{36}$ Ebd. 35.
} 
wo es gemeiniglich um Einsehung der Handlungsbücher, und der gelaufenen Korrespondenz zu thun ist. "37 Mit dieser Übertragung der Besorgung von sehr vielen Angelegenheiten wurde den Wechselgerichten eine weitreichende Zuständigkeit eingeräumt, die jener der späteren Handelsgerichte nicht unähnlich war. ${ }^{38}$ Man kann daher festhalten, dass die Wechselordnung von 1763 einen wichtigen Schritt zur Ausgestaltung der allgemeinen Handelsgerichtsbarkeit darstellte. Dies hatte freilich in anderen Kronländern schon früher begonnen. Mit der „Mercantil und Wechselordnung" von 1722 für die Innerösterreichischen Länder wurde zwar einerseits die Wechselordnung von 1717 weitgehend kopiert, aber für die Hafenstädte Triest [Trieste] und Fiume [Rijeka] bestimmt, dass die Konsulargerichte in Wechselstreitigkeiten und auch in Merkantil- und Navigationssachen Recht zu sprechen hatten, also ebenfalls schon einen frühen Vorläufer der Handelsgerichte bildeten. ${ }^{39}$

Bezüglich der Organisation des Wechselgerichtes bestimmte die neue Wechselordnung Änderungen bezüglich der Eidesleistung, schrieb für den Richter eine Amtsdauer von drei Jahren vor und ließ Richter nach Ablauf ihres Amtes zunächst nur in der zweiten Instanz tätig sein. Der erste Nachfolger des zunächst von der Regierung ernannten Richters hatte aus den Bürgerhändlern zu kommen. Der bereits bekannte alternierende Wechsel fand alle drei Jahre statt, wobei die Richter nicht einfach wieder Beisitzer werden konnten, sondern andere, kompliziertere Regelungen griffen. Die zweite Instanz wurde personell verändert und als dritte Instanz ein Revisorium eingerichtet. ${ }^{40}$

\footnotetext{
${ }_{37}$ Wechselordnung von 1763, Abschnitt Von Wechselund Merkantilgericht erster, anderter und letzter Instanz $\S 1$, in: Theresianisches Gesetzbuch Bd. 4, Nr. 721.

${ }^{38}$ Domin-Petrushevecz, Rechtsgeschichte 82.

${ }^{39}$ WEIGAND, Handelsrechtsgesetzgebung $134 \mathrm{f}$.

${ }^{40}$ Megerle, Ursprüngliche Errichtung $26 \mathrm{f}$.
}

Kaiser Joseph II. dürfte das Wechselgericht in Wien misstrauisch beäugt haben, denn er verfügte die Aufhebung des Wechselgerichtes erster und zweiter Instanz per 1. Mai 1782. Grund dafür dürften Absprachen und zweifelhafte Machenschaften unter den Gerichtspersonen gewesen sein. Der Kaiser wollte die Merkantilprotokolle den politischen Behörden überweisen und die Streitsachen dem bürgerlichen Magistrat der jeweiligen Provinzhauptstadt. Diese hätten die Allgemeine Gerichtsordnung anzuwenden gehabt. Es dürfte zu dieser Aufhebung in Wirklichkeit aber nie gekommen sein und 1786 wurde das Wechselgericht in Wien explizit in seinem Wirkungsbereich bestätigt, aber neu organisiert. Den Vorsitz des Gerichtes hatte der Vizepräsident des niederösterreichischen Landrechtes zu führen. Ihm beigegeben wurden zwei Rechtsgelehrte und je ein Vertreter der drei Handelsklassen, welche alle drei Jahre abwechselten und alle vom Kaiser ernannt werden mussten. ${ }^{41}$ Damit war die Autonomie des Wechselgerichts weitgehend beseitigt. Diese Rechtslage war forthin bestimmend für den Rest des 18 . Jahrhunderts und Ausgangspunkt für Reformen im 19. Jahrhundert.

\section{Korrespondenz:}

Dr. Stefan Wedrac

Österreichische Akademie der Wissenschaften Institut für Neuzeit- und Zeitgeschichtsforschung Strohgasse 45/2d 1030 Wien

stefan.wedrac@oeaw.ac.at

\footnotetext{
${ }^{41}$ BARTSCH, Wiener Gerichte 127; MEGERLE, Ursprüngliche Errichtung 27-29.
} 


\section{Abkürzungen:}

Siehe das allgemeine Abkürzungsverzeichnis: [http://www.rechtsgeschichte.at/files/abk.pdf]

\section{Literatur:}

Joseph Leonard BANNIZA, Vollständige Abhandlung von den sämmtlichen Oesterreichischen Gerichtsstellen (Wien 1767).

Robert BARTSCH, Wiener Gerichte im Vormärz, in: Wiener Ortsausschuß (Hg.), Festschrift zum einunddreißigsten Deutschen Juristentag 3. bis 6. September Wien 1912 (Wien-Leipzig 1912).

Alphons von Domin-PetrusHEvecz, Neuere österreichische Rechtsgeschichte (Wien 1869).

Bogdan DuschKOW-KessiAKOFF, Das Handelsgericht. Ein Beitrag zu Geschichte, Wesen und Wirkung der Handelsgerichte (Greifswald 1912).

Anna KAINRAD, Beiträge zur Geschichte des Wiener Merkantilgerichts (1750-1770) (phil. Diss., Univ. Wien 1953).

Johann Georg MEGERLE, Ursprüngliche Errichtung der k.k. obersten Justizstelle, des k.k. Appellations- und Criminal-Obergerichtes in Österreich ob und unter der Enns, des k.k. n.ö. Landrechtes und des k.k. n.ö. Mercantil- und Wechselgerichtes nebst der Reihenfolge aller diesen GerichtsBehörden vorgestandenen Präsidenten (Wien 1831).

Richard PERGER, Herbert HAUPT, hofbefreite Handwerker, in: Felix CzeIKE (Hg.), Historisches Lexikon Wien. Bd. 3: Ha-La (Wien 1994) 220.

Richard Perger, Niederleger, in: Felix CzeIKe (Hg.), Historisches Lexikon Wien. Bd. 4: Le-Ro (Wien 2004) 398-399.

Max RINTELEN, Das Wiener Merkantilprotokoll, in: ZRG GA 34 (1913) 258-312.

Alfred WALDSTÄTtEN, Staatliche Gerichte in Wien seit Maria Theresia. Beiträge zu ihrer Geschichte. Ein Handbuch (= Forschungen und Beiträge zur Wiener Stadtgeschichte 54, Innsbruck-Wien-Bozen 2011).

Johannes Reinhard WEGELIN, Oesterreichisches Wechsel-Recht oder Wienerische Wechsel-Ordnung Allenthalben mit dienlichen und nöthigen Anmerckungen wie auch vollständigen Registern versehen (Lindau ${ }^{2} 1729$ ).

Arno WeIGAND, Die österreichische Handelsrechtsgesetzgebung vor den großen Kodifikationen. Ein Beitrag zur Geschichte des österreichischen Handels-, Gesellschafts-, Wertpapier-, Banken- und Börserechts vom 17. bis zum 19. Jahrhundert (iur. Diss., Univ. Wien 1997). 